\title{
USO POTENCIAL DE MICROALGAS PARA MITIGAR LOS EFECTOS DE LAS EMISIONES DE DIÓXIDO DE CARBONO
}

\author{
Potential use of microalgae to mitigate the effects of carbon \\ DIOXIDE EMISSIONS
}

Juan Sandoval Herrera*

Diego Rubio Fernández**

Recibido: 14 de agosto de 2017

Aceptado: 11 de septiembre de 2017

\section{Resumen}

Una de las alternativas para controlar o reducir las emisiones de $\mathrm{CO}_{2}$ a la atmósfera es emplear el cultivo de microalgas. Este trabajo presenta una revisión de resultados del uso de microalgas; además, describe las características de las especies y otros factores que pueden influir en el proceso. Finalmente, se realiza una comparación con los resultados obtenidos con especies mayores y con otras técnicas como la adsorción. El objetivo de esta revisión es resaltar el uso potencial de las microalgas para reducir las emisiones de $\mathrm{CO}_{2}$. Se encontró que si se controlan adecuadamente factores como la concentración inicial del gas, la iluminación y la temperatura, el uso de las microalgas es altamente efectivo para capturar y remover el $\mathrm{CO}_{2}$.

Palabras clave: emisiones de $\mathrm{CO}_{2}$, energías alternativas, microalgas, producción de biomasa, contaminación atmosférica.

\section{Abstract}

One of the alternatives to control or reduce $\mathrm{CO}_{2}$ emissions in the atmosphere is to implement microalgae culture. This paper presents a review of the results of the use of microalgae; also, describes the characteristics of the species and other factors that may influence the process. Finally, a comparison with the results obtained with larger species and with different techniques such as adsorption is made. The objective of this review is to highlight the potential use of microalgae to reduce $\mathrm{CO}_{2}$ emissions. It was found that if factors such as initial gas concentration, illumination, and temperature are appropriately controlled, the use of microalgae is highly effective in capturing and removing $\mathrm{CO}_{2}$.

Keywords: $\mathrm{CO}_{2}$ emissions, alternative energies, microalgae, biomass production, atmospheric pollution.

\footnotetext{
* Ingeniero químico, magíster en Formulación y Tecnología del Producto. Docente investigador, Programa de Ingeniería Química, Fundación Universidad de América. Bogotá, Colombia. ORCID:http://orcid.org/0000-0001-89571421. juan.sandoval@profesores.uamerica.edu.co

** Biólogo, magíster en Ciencias, estudiante del Doctorado en Biotecnología (Universidad Nacional de Colombia). Profesor investigador, grupo de investigación BiotecFua, línea de investigación Biotecnología con Algas, Fundación Universidad de América. Bogotá, Colombia. ORCID:http://orcid.org/0000-0003-0760-9567. diego.rubio@profesores.uamerica.edu.co
} 


\section{INTRODUCCIÓN}

Los métodos para reducir o controlar las emisiones de $\mathrm{CO}_{2}$ son muy variados. González-López, Acién, Fernández-Sevilla y Molina (2011) dividen estos métodos según los procesos de captura, almacenamiento, reutilización y técnicas energéticas.

La captura se puede realizar por medios físicos, químicos y biológicos (Zhou et ál., 2017). La decarbonización del combustible previa a su uso (Fiaschi, Gamberi, Bartlett y Griffin, 2005) es una técnica de captura precombustión. El empleo de membranas para separar el $\mathrm{CO}_{2}$ de los gases de salida de un horno es una técnica de captura poscombustión (Ghezel-Ayagh, Jolly, Patel y Steen, 2017). El almacenamiento puede ser geológico, oceánico o en pozos de petróleo vacíos. Dentro de esta categoría se incluye el uso de carbón vegetal para enmienda del suelo (Mulabagal, Baaha, Egieborb y Chenb, 2015). La reutilización incluye el uso del $\mathrm{CO}_{2}$ para ayudar a la extracción mejorada del petróleo o la conversión bioquímica en la producción de biomasa. Rahman et ál. (2017) llaman a esta categoría simplemente "utilización", y recalcan el poco énfasis que se les ha dado a estas técnicas. Una forma de reutilización es el uso biológico como fuente para producir biomasa a partir de algas marinas macroscópicas (Sondak et ál., 2016) o de microalgas para obtener biodiesel. Una de las ventajas de la tecnología de microalgas para reutilizar el $\mathrm{CO}_{2}$ es que se puede acoplar simultáneamente con infraestructuras existentes de generación de energía y tratamiento de aguas (Kumar et ál., 2010). Las técnicas energéticas incluyen la mejora de la eficiencia en las centrales térmicas, el empleo de energías alternativas, el reciclado de gases de chimenea que normalmente se queman (Khanipour, Mirvakili, Bakhtyari, Farniaeiy Reza, 2017) o la reforestación (IPCC, 2015).

En este artículo se definen los objetivos del proceso de fijación de $\mathrm{CO}_{2}$ por medio de microalgas, mostrando algunos resultados encontrados a nivel laboratorio, planta piloto y escala industrial. Se analizan algunos factores que influyen en el proceso y se comparan los resultados destacados de diferentes especies de microalgas, con los obtenidos por otras especies de orden mayor y por otras técnicas.

\section{DesarRollo del tema}

A nivel industrial se busca que la capacidad de fijación de $\mathrm{CO}_{2}$ (así como la productividad del cultivo) sea alta. Igualmente, se busca lograr dos objetivos: la protección del medio ambiente por la reducción de la concentración atmosférica de este gas de efecto invernadero y la posible producción de sustancias valiosas (biodiesel, principalmente) a partir de la biomasa cultivada.

\section{Biofijación}

Se define como la capacidad de almacenamiento de $\mathrm{CO}_{2}$ que tiene un cultivo de microalgas. Según González-López et ál. (2011), una hectárea de plantas puede fijar 17 toneladas de dióxido de carbono por año; igualmente, la misma hectárea de microalgas puede fijar hasta 75 toneladas en el mismo año. De acuerdo con Zhou et ál. (2017), se fijan 183 toneladas de $\mathrm{CO}_{2}$ por cada 100 toneladas de biomasa producida. Para estudiar esta capacidad de las microalgas, se realizan ensayos a nivel laboratorio en los que se evalúa la masa de $\mathrm{CO}_{2}$ fijada por volumen y tiempo de cultivo. No todas las especies de microalgas tienen la misma capacidad de fijación de $\mathrm{CO}_{2}$; por otra parte, en una misma especie se observan resultados diferentes (ver anexo 1 ) dependiendo de las condiciones del proceso (esto se analizará más adelante). 


\section{Productividad}

Relaciona la cantidad de biomasa que se obtiene en un tiempo determinado por volumen de cultivo. Se calcula por medio de la relación entre la diferencia de la concentración celular $\left(\mathrm{g} \mathrm{L}^{-1}\right)$ en un tiempo dado menos la concentración inicial y el tiempo. Sankar et ál. (2011) midieron la productividad para tres especies (Spirulina platensis, Calothrix sp. y Chlorella minutissima) en matraces agitados, en los que optimizaron las condiciones de $\mathrm{pH}$, fotoperiodo, intensidad lumínica y tipo de agitador, con una concentración inicial de $15 \%$ en volumen de $\mathrm{CO}_{2}$ en la mezcla con aire del cultivo. Después de 138 horas de cultivo, la investigación reportó $0.88 \mathrm{~g}$ de biomasa seca por litro de medio. Chiu et ál. (2009) determinaron la productividad total de biomasa de Nannochloropsis oculata a concentraciones volumétricas iniciales de 2, 5,10 y $15 \%$ de $\mathrm{CO}_{2}$, hallando que disminuía desde 0.480 hasta $0.372 \mathrm{~g} \mathrm{~L}^{-1} \mathrm{~d}^{-1}$. Según Yun et ál (2016), Acutodesmus obliquus presentó una productividad de $0.153 \mathrm{~g} \mathrm{~L}^{-1} \mathrm{~d}^{-1}$ al cabo de 3 días, bajo una concentración volumétrica inicial de $14 \%$ de $\mathrm{CO}_{2}$. En el estudio de Ramaraj, Tsai y Chen (2014) se reportó una productividad de $0.074 \mathrm{~g} \mathrm{~L}^{-1} \mathrm{~d}^{-1}$ con un sistema de cultivo de mezcla de algas en un fotobiorreactor de 4 L. Kaštánek et ál. (2010) reportaron $0.4 \mathrm{gL}^{-1} \mathrm{~d}^{-1}$ de productividad máxima para Chlorella vulgaris en un fotobiorreactor de placa plana de $1.5 \mathrm{~L}$ alimentado con aire enriquecido con $2 \%$ en volumen de $\mathrm{CO}_{2}$ y bajo luz natural. Para la misma especie, Kin-Chung, Chi-Chung, On-Kit y Ho-Man (2013) registraron $0.343 \mathrm{~g} \mathrm{~L}^{-1} \mathrm{~d}^{-1}$ después de 10 días de cultivo en reactores cónicos de $1 \mathrm{~L}$ a temperatura de $26{ }^{\circ} \mathrm{C}$,

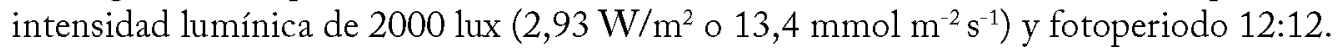

\section{Factores que afectan la productividad y la capacidad de fijación}

Como se ha mostrado en la sección anterior, los resultados del proceso de biofijación microalgal de $\mathrm{CO}_{2}$ dependen de múltiples factores, como concentración inicial de $\mathrm{CO}_{2}$, luz y diseño del reactor, entre otros. En esta sección se describirán algunos de estos factores y se esbozará la relación de estos con los resultados de productividad y biofijación.

\section{Tolerancia de la especie y concentración inicial de $\mathrm{CO}_{2}$}

La tolerancia de la especie es la concentración volumétrica máxima de $\mathrm{CO}_{2}$ que puede soportar una microalga para crecer. Un primer paso en un proyecto de biofijación de $\mathrm{CO}_{2}$ es la selección de la especie; aquí se busca la microalga que mayor tolerancia tenga a nivel de laboratorio. En muchos casos se va aumentando la concentración inicial de $\mathrm{CO}_{2}$ en el aire enriquecido con este gas, y se evalúa la productividad de biomasa al cabo de cierto tiempo. Yun, Lee, Park, Lee y Yang (1997) probaron la productividad variando la concentración de $\mathrm{CO}_{2}$ en al aire desde $5 \%$ hasta $15 \%$ en volumen para un cultivo de Chlorella vulgaris; según los autores, si la microalga se adapta previamente a un 5\%, puede resistir el incremento posterior a $15 \%$ (y posiblemente valores mayores), lo que aumenta la capacidad de fijación de $\mathrm{CO}_{2}$. Gaikwad et ál. (2016) encontraron que Scenedesmus sp. tolera $80 \%$ en volumen de este gas; Chlorella sp., $40 \%$, y la Nannochloris sp., apenas un $15 \%$. Este mismo porcentaje fue encontrado para la Chlorella vulgaris (Kin-Chung et ál., 2013), con una productividad de $0.203 \mathrm{~g} \mathrm{~L}^{-1} \mathrm{~d}^{-1}$ después de 10 días. La selección de la especie es tan compleja que incluso Han, Li, Miao y Yu (2012) idearon un sistema giratorio automatizado para realizar esta etapa. Los valores de tolerancia al $\mathrm{CO}_{2}$ para una misma especie pueden variar según el investigador, debido al diseño del reactor o la intensidad y calidad de la luz suministrada. Así, por ejemplo, Vijendren, Uemura, Yusup y Osman (2014), en la revisión de resultados para la Nannochloropsis sp., reportaron que $1.5 \%$ de $\mathrm{CO}_{2}$ produce $0.24 \mathrm{~g} \mathrm{~L}^{-1} \mathrm{~d}^{-1} ; 2 \%$ de $\mathrm{CO}_{2}, 0.497 \mathrm{~g} \mathrm{~L}^{-1} \mathrm{~d}^{-1}$, y $3 \%$ de $\mathrm{CO}_{2}, 0,3 \mathrm{~g} \mathrm{~L}^{-1} \mathrm{~d}^{-1}$. En cada caso se emplearon diferente tipo de reactor (panel plano, cilíndrico

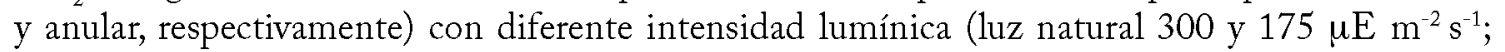


respectivamente). Por otra parte, la tolerancia al $\mathrm{CO}_{2}$ varía cuando el proceso se escala a nivel industrial (García, 2014).

Los mecanismos biológicos por los cuales cada especie se adapta a diferentes niveles de concentración de $\mathrm{CO}_{2}$ en condiciones ambientales diversas, como las que se pueden presentar a escala industrial, han sido estudiados por Giordano, Beardall y Raven (2005), Young y Beardall (2005), Moroney y Ynalvez (2007) y Collins, Sueltemeyer y Bell (2006), entre otros.

\section{Diseño del reactor}

Puede ser de tipo abierto (lagunas o estanques) o de tipo cerrado: panel plano vertical, tubular horizontal, tubular vertical, columna vertical, entre otros. Fan et ál. (2007) diseñaron un sistema en continuo de fotobiorreactor de membrana a nivel laboratorio, en el que optimizaron las condiciones del cultivo de Chlorella vulgaris. Alcanzaron una mayor fijación de $\mathrm{CO}_{2}$ en este reactor $(0.275$ $\mathrm{g} \mathrm{L}^{-1} \mathrm{~h}^{-1}$ o $0.011 \mathrm{~g} \mathrm{~L}^{-1} \mathrm{~d}^{-1}$ ) comparado con otros diseños (columna de burbujeo, Air lift y contactor de membrana). Lutzu (2012) evaluó primero el uso potencial de Chlorella vulgaris en reactores por lotes; luego escaló la producción a fotobiorreactores tubulares continuos con miras a la producción de bioaceites. García (2014) realizó el cultivo de dos especies (Scenedesmus vacuolatus y Chlorella vulgaris) a nivel laboratorio en reactor continuo tipo fotoquimiostato; posteriormente, evaluó el cultivo a escala industrial en estanque y reactor plano vertical, encontrando que la $C$. vulgaris resistía mejor el cultivo a la intemperie (fue capaz de fijar $\mathrm{CO}_{2}$ para la producción de almidones) y que el mejor diseño por factores económicos y rendimiento del cultivo era el tipo vertical.

\section{Intensidad y calidad de la luz}

La intensidad luminica depende de si es luz natural o artificial y qué tan alto sea el flujo lumínico. Así, por ejemplo, Li et ál. (2012) estudiaron el efecto de la intensidad lumínica sobre el crecimiento de Chlorella kessleri y Chlorella protothecoide en agua residual proveniente del proceso de deshidratación de lodos activados de una planta de tratamiento de agua residual domiciliaria, encontrando que con intensidades desde 0 hasta $120 \mu \mathrm{mol} \mathrm{m}^{-2} \mathrm{~s}^{-1}$ se presentaba crecimiento para C. kessleri. No obstante, verificaron que con intensidades superiores había fotoinhibición. Al parecer, con Chlorella protothecoide después de $30 \mathrm{mmol} \mu^{-2} \mathrm{~s}^{-1}$ se retrasaba la acumulación de biomasa microalgal.

Naderi, Tadé y Znad (2015) probaron cinco intensidades (30, 50, 100, 185 y $300 \mathrm{mmol} \mathrm{m}^{-2} \mathrm{~s}^{-1}$ ) con Chlorella vulgaris; observaron fotolimitación para intensidades inferiores a $50 \mu \mathrm{mol} \mathrm{m} \mathrm{m}^{-2} \mathrm{~s}^{-1} \mathrm{y}$ fotoinhibición para intensidades superiores a $185 \mathrm{mmol} \mathrm{m}^{-2} \mathrm{~s}^{-1}$. La máxima biofijación de $\mathrm{CO}_{2}$ fue $0.45 \mathrm{~g} \mathrm{~L}^{-1} \mathrm{~d}^{-1}$ (ver anexo 1) con intensidad de $100 \mathrm{mmol} \mathrm{m}^{-2} \mathrm{~s}^{-1}$. Mejía, Colmenares y Voroney (2013) ensayaron con tres diferentes tipos de luz emitida por diodos (LED): roja, con longitud de onda de $620-625 \mathrm{~nm}$ e intensidad de $1345 \mu \mathrm{mol} \mathrm{m}{ }^{-2} \mathrm{~s}^{-1}$; azul, $425-430 \mathrm{~nm}$ y $2143 \mu \mathrm{mol} \mathrm{m}^{-2} \mathrm{~s}^{-1}$, y blanca, 380-760 nm y $1838 \mu \mathrm{mol} \mathrm{m}^{-2} \mathrm{~s}^{-1}$. Esta intensidad disminuye con la distancia; por su parte, los diodos emisores estaban a $7 \mathrm{~mm}$ de la suspensión algal para minimizar la fotoinhibición. Los autores encontraron que con una concentración inicial de $8.5 \%$ de $\mathrm{CO}_{2}$ en aire la productividad alcanza su máximo nivel $\left(1.6 \mathrm{gL}^{-1} \mathrm{~d}^{-1}\right)$ con luz azul, mientras que con luz roja la máxima productividad se alcanzó con una concentración de $\mathrm{CO}_{2}$ inicial de $3.7 \%\left(0.57 \mathrm{~g} \mathrm{~L}^{-1} \mathrm{~d}^{-1}\right)$.

$\mathrm{Hu}$, Zhou y Liu (2016) desarrollaron una celda de captura microbial de carbón tipo air-lift (ALMCC, por sus siglas en inglés) con el fin de encontrar los valores óptimos de intensidad lumínica para Chlorella vulgaris. En el estudio se determinó que a $8.9 \mathrm{~W} \mathrm{~m}^{-2}$ (aproximadamente 40.6

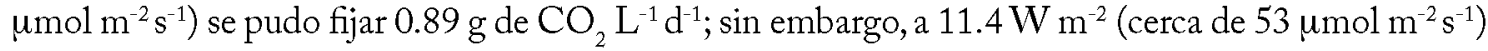


la fijación disminuía a $0.78 \mathrm{~g} \mathrm{~L}^{-1} \mathrm{~d}^{-1}$. Teniendo en cuenta estos resultados, ${ }^{1}$ se podría decir que a pesar de que hay una fuerte dependencia del crecimiento microalgal con la intensidad lumínica, no se puede concluir cuantitativamente acerca de la relación de esta con la capacidad de fijación de $\mathrm{CO}_{2}$.

\section{Otros factores}

El fotoperiodo (Wijanarko, Dianursanti, Witarto y Soemantojo, 2004; Ho, Chen y Chang, 2010) afecta de forma directamente proporcional la capacidad de fijación de $\mathrm{CO}_{2}$. Por su parte, Colla, Reinehr, Reichert y Vieira (2017) evaluaron la influencia de la temperatura en la productividad de Spirulina platensis. La frecuencia de suministro de $\mathrm{CO}_{2}$ fue analizada por Kin-Chung et ál. (2013) para el caso de Chlorella vulgaris. La velocidad de flujo de mezcla gaseosa alimentada, evaluada por Fan et ál. (2007), afecta de forma inversamente proporcional a la capacidad de fijación de $\mathrm{CO}_{2}$. Todos estos son factores que se deben tener en cuenta a la hora de escalar cualquier proceso de biofijación microbiológica a nivel industrial.

\section{Comparación de resultados}

\section{Entre diferentes especies de microalgas}

En el anexo 1 se muestran algunos resultados de fijación y productividad a escala laboratorio con diferentes especies, así como se destacan las condiciones más relevantes en las que se obtuvieron los resultados. Como se observa, la especie con los mejores resultados fue Scenedesmus obliquus, que toleró hasta un $10 \%$ de $\mathrm{CO}_{2}$ y consiguió remover $0.55 \mathrm{~g} \mathrm{~L}^{-1} \mathrm{~d}^{-1}$.

\section{Con respecto a otras técnicas}

Cuando se emplean adsorbentes físicos, la capacidad de fijación se expresa en unidades de mmol $\mathrm{CO}_{2} / \mathrm{g}$ adsorbente; por ejemplo, el carbón activado de hueso de aceituna tratado con $\mathrm{KOH}$ adsorbe $5.6 \mathrm{mmol}$ de $\mathrm{CO}_{2}$ por gramo de material adsorbente (Moussa et ál., 2017). Las nanopartículas mesoporosas de óxido de magnesio adsorben $1.34 \mathrm{mmol}$ de $\mathrm{CO}_{2}$ por gramo de material adsorbente (Hiremath, Shavi y Seo, 2017). Las mezclas acuosas de monoetanol amina y glicerol adsorben $0.886 \mathrm{~mol} \mathrm{de} \mathrm{CO}_{2}$ por mol de amina a $1500 \mathrm{kPa}$ (Shamiri et ál., 2016). Tsai, Chen y Ramaraj (2017) compararon la capacidad de fijación de $\mathrm{CO}_{2}$ de microalgas a escala industrial $\left(23.86 \mathrm{~g} \mathrm{~m}^{-2} \mathrm{~d}^{-1}\right)$ con la de plantas maderables $\left(4.80 \mathrm{~g} \mathrm{~m}^{-2} \mathrm{~d}^{-1}\right)$ y plantas no maderables $\left(0 \mathrm{mg} \mathrm{m}^{-2} \mathrm{~d}^{-1}\right)$.

Teniendo en cuenta la densidad promedio de cultivo de microalga, similar a la del agua, se puede calcular el rendimiento en unidades de $\mathrm{g} \mathrm{CO}_{2} / \mathrm{g}$ cultivo. De manera que, según Fulke Krishnamurthi, Giripunje, Saravana y Chakrabarti (2015), el máximo $1.8 \mathrm{~g} / \mathrm{g}$ por un día de proceso sería mayor al obtenido por Moussa et ál. (2017), de $5.6 \mathrm{mmol} / \mathrm{g}=0,246 \mathrm{~g} / \mathrm{g}$ adsorbente, y más aún que el de Hiremath et ál. (2017), de $1.34 \mathrm{mmol} / \mathrm{g}=0.059 \mathrm{~g} / \mathrm{g}$ adsorbente (ver anexo 1).

\footnotetext{
${ }^{1}$ Al respecto, Martínez (2012) afirma: "los valores de fotosaturación (fotoinhibición) para las especies no son muy concisos" (p. 5).
} 


\section{Tendencias actuales}

Existen algunos trabajos que buscan acoplar el cultivo de microalga con otro tipo de tecnología de captura de $\mathrm{CO}_{2}$, como, por ejemplo, los biodepuradores o torres de adsorción, que incorporen microalgas como parte de biofiltros o biomembranas de adsorción (Jeong, Gillis y Hwang, 2003). También se observa el uso de fotobiorreactores in situ con membranas (Wiley, 2013). Finalmente, el estudio del mecanismo interno celular por el cual el $\mathrm{CO}_{2}$ es asimilado en cada microalga es un tema que está en continuo desarrollo (ver, por ejemplo, Singh, Sundaram, Sinha, Rahman y Kapur, 2016; Goli et ál., 2016).

\section{Conclusiones}

La capacidad de fijación de $\mathrm{CO}_{2}$ obtenida por las microalgas es superior a la de macroalgas y plantas de orden superior (Tsai et ál., 2017) y a la de los adsorbentes físicos estudiados (Shamiri et ál., 2016; Moussa et ál., 2017; Hiremath et ál., 2017). Según los trabajos analizados en esta revisión (ver anexo 1), los géneros de microalga que toleran mayores concentraciones de $\mathrm{CO}_{2}$ son Scenedesmus, Spirulina (cianobacteria) y Chlorella. Los factores que se relacionan con el rendimiento en el proceso son: tolerancia de la especie a la concentración de $\mathrm{CO}_{2}$, diseño del reactor, intensidad y calidad de la luz, fotoperiodo, velocidad de flujo, concentración de $\mathrm{CO}_{2}$ inicial, entre otros. Se consigue mayor rendimiento a menor concentración inicial de $\mathrm{CO}_{2}$ y mayor intensidad lumínica, hasta cierto valor de fotoinhibición; pero en cada especie los valores óptimos de estos parámetros son diferentes y dependen del tipo de reactor. Finalmente, de acuerdo con esta revisión, se observa que pocas especies pueden soportar emisiones de gases de chimenea sin purificar.

\section{RefERENCIAS}

Adinurani, P., Setyobudi, R., Wahono, S., Mel, M., Nindita, A., Purbajanti, E., Harsono, S., Malala, A., y Sasmito, A. (2016). Carbon dioxide capture efficiency using algae biological absorbent and solid adsorbent for biogas purification. Jurnal Teknologi, 78, 175-178

Chiu, S., Kao, C., Chen, C., Kuan, T., Ong, S., y Lin, C. (2008). Reduction of CO2 by a highdensity culture of Chlorella sp. in a semicontinuous photobioreactor. Bioresource technology, 99(9), 3389-3396.

Chiu, S., Kao, C., Tsai, M., Ong, S., Chen, C., y Lin, C. (2009). Lipid accumulation and $\mathrm{CO}_{2}$ utilization of Nannochloropsis oculata in response to $\mathrm{CO}_{2}$ aeration. Bioresource technology, 100(2), 833-838.

Colla, L., Reinehr, C., Reichert, C. y Vieira, J. (2017). Production of biomass and nutraceutical compounds by Spirulina platensis under different temperature and nitrogen regimes. Bioresource technology, 98(7),1489-93

Collins, S., Sueltemeyer, D., y Bell, G. (2006). Changes in C uptake in populations of Chlamydomonas reinhardtii selected at high $\mathrm{CO}_{2}$. Plant, cell y environment, 29(9), 1812-1819.

Fan, L., Zhang, Y., Cheng, L., Zhang, L., Tang, D., y Chen, H. (2007). Optimization of carbon dioxide fixation by Chlorella vulgaris cultivated in a membrane-photobioreactor. Chemical engineering y technology, 30(8), 1094-1099.

Fiaschi, D., Gamberi, F., Bartlett, M. y Griffin, T., (2005). The air membrane-ATR integrated gas turbine power cycle: A method for producing electricity with low $\mathrm{CO}_{2}$ emissions. Energy Conversion and Management, 46(15), 2514-2529 
Fulke, A., Krishnamurthi, K., Giripunje, M., Saravana, S. y Chakrabarti, T. (2015). Biosequestration of carbon dioxide, biomass, calorific value and biodiesel precursors production using a novel flask culture photobioreactor. Biomass and bioenergy, 72, $136-142$

Gaikwad, R, Gudadhe, M. y Bhagat, S. (2016). Carbon Dioxide Capture, Tolerance and Sequestration Using Microalgae- A Review. International Journal of Pharmaceutical, Chemical and Biological Sciences, 6(3), 345-349

García, R. (2014). Producción de biomasa de microalgas rica en carbohidratos acoplada a la eliminación fotosintética de $\mathrm{CO}_{2}$ (tesis doctoral). Instituto de Bioquímica Vegetal y Fotosíntesis, Universidad de Sevilla, Sevilla, España.

Ghezel-Ayagh, H., Jolly, S., Patel, D., y Steen, W. (2017). Electrochemical membrane technology for carbon dioxide capture from flue gas. Energy Procedia, 108, 2-9.

Giordano, M., Beardall, J. y Raven, J. (2005). $\mathrm{CO}_{2}$ concentrating mechanisms in algae: mechanisms, environmental modulation, and evolution. Annual reviews of plant biology, 56, 99-131. doi: 10.1146/ annurev. arplant.56.032604.144052

Goli, A., Shamiri, A., Talaiekhozani, A., Eshtiaghi, N., Aghamohammadi, N., y Aroua, M. K. (2016). An overview of biological processes and their potential for $\mathrm{CO}_{2}$ capture. Journal of environmental management, 183, 41-58.

González-López, C., Acién, F., Fernández-Sevilla, J., Molina, E. (2011). Uso de microalgas como alternativa a las tecnologías disponibles de mitigación de emisiones antropogénicas de $\mathrm{CO}_{2}$. Revista Latinoamericana de Biotecnología Ambiental Algal, 2(2), 93-106.

Greque, M., y Vieira, J. (2007a). Biofixation of carbon dioxide by Spirulina sp. and Scenedesmus obliquus cultivated in a three-stage serial tubular photobioreactor. Journal of Biotechnology, 129(3), 439-445. doi.org/10.1016/j.jbiotec.2007.01.009

Greque, M., y Vieira, J. (2007b). Carbon dioxide fixation by Chlorella kessleri, C. vulgaris, Scenedesmus obliquus and Spirulina sp. Cultivated in flasks and vertical tubular photobioreactors. Biotechnology letters, 29(9), 1349-1352. doi 10.1007/s10529-007-9394-6

Han, W., Li, Ch., Miao, X. y Yu, G. (2012). A novel miniature culture system to screen $\mathrm{CO}_{2}-$ sequestering microalgae. Energies, 5(11), 4372-4389. doi:10.3390/en5114372

Hiremath, V., Shavi, R., y Seo, J. (2017). Mesoporous magnesium oxide nanoparticles derived via complexation-combustion for enhanced performance in carbon dioxide capture. Journal of Colloid and Interface Science, 498, 55-63

Ho, S-H., Chen, W-M., y Chang, J-S. (2017). Scenedesmus obliquus CNW-N as a potential candidate for $\mathrm{CO}_{2}$ mitigation and biodiesel production. Bioresource technology, 101(22), 87258730 .

Hu, X., Zhou, J., y Liu, B. (2016). Effect of algal species and light intensity on the performance of an air-lift-type microbial carbon capture cell with an algae-assisted cathode. The Royal Society of Chemistry, 6(30), 25094-25100.

IPCC. (2015). Cambio climático 2014: informe de sintesis. Contribución de los grupos de trabajo i, ii $y$ iii al quinto informe de evaluación del grupo Intergubernamental de Expertos sobre el Cambio Climático. Ginebra, Suiza: IPCC. 
Jeong, M., Gillis, J. y Hwang, J-Y. (2003). Carbon dioxide mitigation by microalgal photosynthesis. Bulletin of Korean Chemistry Society, 24(12), 1763-1766

Kaštánek, F., Šabata, S., Šolcová, O., Maléterová, Y., Kaštánek, P., Brányiková, I., Kuthan, K., y Zachleder, V. (2010). In-field experimental verification of cultivation of microalgae Chlorella sp. using the flue gas from a cogeneration unit as a source of carbon dioxide. Waste Management y Research, 28(11), 961-966.

Khanipour, M., Mirvakili, A., Bakhtyari, A., Farniaei, M., y Reza, M. (2017). Enhancement of synthesis gas and methanol production by flare gas recovery utilizing a membrane based separation process. Fuel Processing Technology, 166, 186-201. dx.doi.org/10.1016/j.fuproc.2017.06.008

Kin-Chung, W., Chi-Chung, L., On-Kit, H., y Ho-Man, Y. (2013). A Study on Algal Growth Behavior under different Sparging Period of $\mathrm{CO}_{2}$ Supplementation. Ponecia presentada en 1 st International Conference on Beneficial Uses of Algal Biomass, ICBUAB, Hong Kong.

Kumar, A., Ergas, S., Yuan, X., Sahu, A., Zhang, Q. Dewulf, J., Malcata, F., y van Langenhove, H. (2010). Enhanced $\mathrm{CO}_{2}$ fixation and biofuel production via microalgae: recent developments and future directions. Trends in biotechnology, 28(7), 371-380.

Li, Y., Zhou, W., Hu, B., Min, M., Chen, P., y Ruan, R. (2012). Effect of light intensity on algal biomass accumulation and biodiesel production for mixotrophic strains Chlorella kessleri and Chlorella protothecoide cultivated in highly concentrated municipal wastewater. Biotechnology and bioengineering, 109(9), 2222-2229.

Lutzu, G. (2012). Analysis of the growth of microalgae in batch and semi-batch photobioreactors (disertación doctoral). Universidad de Cagliari, Cagliari Italia.

Martínez, J. (2012). Modelo de biomasa algal para la captura de $\mathrm{CO}_{2}$ y su desarrollo en un software de evaluación (trabajo de maestría). Máster en Investigación en Ingeniería para el Desarrollo Agroforestal, Escuela de ingenierías agrarias, Universidad de Valladolid, Valladolid, España.

Mejía, S., Colmenares, G., y Voroney, P. (2013). Effect of carbon dioxide concentration on the growth response of Chlorella vulgaris under four different led illuminations. International journal of biotechnology for wellness industries, 2(3), 125-131.

Moroney, J. y Ynalvez, R. (2007). Proposed Carbon Dioxide Concentrating Mechanism in Chlamydomonas reinhardtii. Eukaryotic Cell, 6(8), 1251-1259. doi:10.1128/EC.00064-07

Moussa, M., Bader, N., Querejeta, N., Durán, I., Pevida, C., y Ouederni, A. (2017). Toward sustainable hydrogen storage and carbon dioxide capture in post-combustion conditions. Journal of Environmental Chemical Engineering, 5(2), 1628-1637.

Mulabagala, V., Baaha, D., Egieborb, N., y Chenb, W. (2015). Biochar from Biomass: A strategy for carbon dioxide sequestration, soil amendment, power generation, and $\mathrm{CO}_{2}$ utilization. En W. Chen, T. Suzuki, Toshio, M. Lackner (Eds.), Handbook of Climate Change Mitigation and Adaptation (pp. 1937-1974). Nueva York: Springer.

Naderi, G., Tade, M. y Znad, H. (2015). Modified photobioreactor for biofixation of carbon dioxide by Chlorella vulgaris at different light intensities. Chemical engineering and technology, 38(8), 1371-1379

Rahman, F., Aziz, M., Saidur, R., Bakar, W., Hainin, M., Putrajaya, R., y Hassan, N. (2017). Pollution to solution: Capture and sequestration of carbon dioxide $\left(\mathrm{CO}_{2}\right)$ and its utilization 
as a renewable energy source for a sustainable future. Renewable and Sustainable Energy Reviews, 71, 112-126.

Ramaraj, R., Tsai, D. y Chen, P. (2014). Freshwater microalgae niche of air carbon dioxide mitigation. Ecological Engineering, 68, 47-52

Sankar, V., Daniel, D. y Krastanov, A., (2011). Carbon dioxide fixation by Chlorella minutissima batch cultures in a stirred tank biorreactor. Biotechnology y Biotechnological Equipment, 25(3), 2468-2476. doi: 10.5504/BBEQ.2011.0058

Shabani, M., Sayadi, M., y Rezaei, M. (2016). $\mathrm{CO}_{2}$ bio-sequestration by Chlorella vulgaris and Spirulina platensis in response to different levels of salinity and $\mathrm{CO}_{2}$ Proceedings of the International Academy of Ecology and Environmental Sciences, 6(2), 53-61

Shamiri, A., Shafeeyan, M., Tee, H., Leo, C., Aroua, M., y Aghamohammadi, N. (2016). Absorption of $\mathrm{CO}_{2}$ into aqueous mixtures of glycerol and monoethanolamine. Journal of Natural Gas Science and Engineering, 35, 605-613.

Singh, S., Rahman, A., Dixit, K., Nath, A. y Sundaram, S. (2015). Evaluation of promising algal strains for sustainable exploitation coupled with $\mathrm{CO}_{2}$ fixation. Environmental technology, 37(5), 613-622. doi: 10.1080/09593330.2015.1075599

Singh, S., Sundaram, S., Sinha, S., Rahman, A. y Kapur, S. (2016). Recent advances in $\mathrm{CO}_{2}$ uptake and fixation mechanism of cyanobacteria and microalgae. Critical reviews in environmental science and technology, 46(16), 1297-1323. http://dx.doi.org/10.1080/10643389.2016.1217911

Sondak, C., Ang, P., Beardall, J., Bellgrove, A., Boo, S., Gerung, G., Hepburn, C., Hong, D., Hu, Z., Kawai, H., Largo, D., Lee, J., Lim, P., Mayakun, J., Nelson, W., Hyun, J., Phang, S., Sahoo, D., Peerapornpis, YangIk, Y., y Chung, I. (2016). Carbon dioxide mitigation potential of seaweed aquaculture beds (SABs). Journal of Applied Phycology, 1-11. https://doi. org/10.1007/s10811-016-1022-1

Sydney, E., Sturm, W., de Carvalho, J., Thomaz-Soccol, V., Larroche, C., Pandey, A., y Soccol, C. (2010). Potential carbon dioxide fixation by industrially important microalgae. Bioresource technology, 101(15), 5892-5896.

Tang, D., Han, W., Li, P., Miao, X., y Zhong, J. (2011). CO 2 biofixation and fatty acid composition of Scenedesmus obliquus and Chlorella pyrenoidosa in response to different $\mathrm{CO}_{2}$ levels. Bioresource Technology, 102(3), 3071-3076

Tsai, D., Chen, P. y Ramaraj, R. (2017). The potential of carbon dioxide capture and sequestration with algae. Ecological engineering, 98, 17-23.

Velásquez, A., Alza, V., Flores, C., Gutiérrez, L., Sánchez, J., Bernabé, P., y Bernabé, N. (2014). Escalamiento de fotobiorreactor solar secuestrante de $\mathrm{CO}_{2}$ de gases de combustión optimizando producción de "espirulina". SCIÉNDO, 15(1), 7-21.

Vijendren, K., Uemura, Y., Yusup, S. y Osman, N. (2014). Aspects of carbon dioxide mitigation by Nannochloropsis oculata cultured in a photobioreactor. Applied mechanics and materials. 625 , 775-779. doi:10.4028/www.scientific.net/AMM.625.775

Wijanarko, A., Dianursanti, Witarto, A.B. y Soemantojo, R.W. (2004). Effect of photoperiodicity on $\mathrm{CO}_{2}$ fixation by Chlorella vulgaris buitenzorg in bubble column photobioreactor for food supplement production. Makara, teknologi, 8(2), 35-43 
Wiley, P. (2013). Microalgae cultivation using offshore membrane enclosures for growing algae (OME$G A)$ (disertación doctoral). University of California, California, EE. UU.

Young, E., y Beardall, J. (2005). Modulation of photosynthesis and inorganic carbon acquisition in a marine microalga by nitrogen, iron, and light availability. Canadian Journal of Botany, 83(7), 917-928.

Yun, H-S., Ji, M-K., Park, Y-T., Salama, E-S y Choi, J. (2016). Microalga, Acutodesmus obliquus KGE 30 as a potential candidate for $\mathrm{CO}_{2}$ mitigation and biodiesel production. Environmental Science and Pollution Research 23(17), 17831-17839. doi 10.1007/s11356-016-6971-z

Yun, Y-S., Lee, S., Park, J.M., Lee, C. y Yang. J-W. (1997). Carbon Dioxide Fixation by Algal Cultivation Using Wastewater Nutrients. Journal of Chemistry Technology and Biotechnology. 69(4), 451-455

Zhou, W., Wang, J., Chen, P., Ji, C., Kang, Q., Lu, B., Li, K., Liu, J., y Ruan, R. (2017). Bio-mitigation of carbon dioxide using microalgal systems: Advances and perspectives. Renewable and Sustainable Energy Reviews, 76, 1163-1175. 
Anexo 1. Resultados de fijación de $\mathrm{CO}_{2}$ a escala laboratorio para varias especies de microalgas

\begin{tabular}{|c|c|c|c|c|}
\hline Especie algal & $\begin{array}{c}\% \text { entrada de } \\
\mathrm{CO}_{2} \\
\end{array}$ & Fijación de $\mathrm{CO}_{2}$ & Detalles & Referencia \\
\hline Spirulina sp. & 0.04 & $99.90 \%$ & VTP 4L & Greque y Vieira (2007a) \\
\hline Spirulina sp. & 6 & $0.39 \mathrm{gL}^{-1} \mathrm{~d}^{-1}$ & CPBR 2L & Greque y Vieira (2007b) \\
\hline Spirulina sp. & 6 & $27-38 \%$ & CPBR 2L & Greque y Vieira (2007b) \\
\hline $\begin{array}{l}\text { Spirulina sp. } \\
\text { (A. Jenneri) }\end{array}$ & $11.70 \%$ & $58.00 \%$ & TPBR $44 \mathrm{~L} 96$ horas & Velásquez et ál (2014) \\
\hline Scenedesmus obliquus & 6 & $7-14 \%$ & CPBR 2L & Greque y Vieira (2007b) \\
\hline Scenedesmus obliquus & 0.04 & $88.00 \%$ & VTP 4L & Greque y Vieira (2007b) \\
\hline Scenedesmus obliquus & 6 & $2.00 \%$ & CPBR 2L & Greque y Vieira (2007b) \\
\hline Scenedesmus obliquus & 10 & $0.55 \mathrm{gL}^{-1} \mathrm{~d}^{-1}$ & PBR $1 \mathrm{~L}$, Fotoperiodo $24: 0 . \mathrm{I}_{0}: 60 \mu \mathrm{mol} \mathrm{m} \mathrm{-}^{-2} \mathrm{~s}^{-1}$ & Ho, Chen y Chang (2010) \\
\hline Scenedesmus obliquus & 7.5 & $1.8 \mathrm{gL}^{-1} \mathrm{~d}^{-1}$ & $250 \mathrm{~mL}$ Erlenmeyer & $\begin{array}{l}\text { Fulke, Krishnamurthi, Giripunje, Saravana y } \\
\text { Chakrabarti (2015) }\end{array}$ \\
\hline Scenedesmus obliquus & 10 & $0.29 \mathrm{gL}^{-1} \mathrm{~d}^{-1}$ & Erlenmeyer $800 \mathrm{~mL} 25^{\circ} \mathrm{C} . \mathrm{I}_{0}: 180 \mu \mathrm{mol} \mathrm{m}^{2} \mathrm{~s}^{-1}$ & Tang, Han, Li, Miao y Zhong (2011) \\
\hline Scenedesmus sp. & & $14-22 \%$ & Emisiones directamente de planta industrial & Adinurani et al. (2016) \\
\hline Chlorella sp. & 2 & $58 \%$ & CPBR $800 \mathrm{~mL}$ & Chiu et al. (2008) \\
\hline Chlorella sp. & 10 & $0,125 \mathrm{gL}^{-1} \mathrm{~d}^{-1}$ & Sistema ALMCC. Cátodo $500 \mathrm{~mL}$ & Hu, Zhou y Liu (2016) \\
\hline Chlorella sp. & 6 & $0.45 \mathrm{gL}^{-1} \mathrm{~d}^{-1}$ & CPBR $2,25 \mathrm{~L} 27^{\circ} \mathrm{C} 4320$ lux. Fotoperiodo $14: 10$ & Singh, Rahman, Dixit, Nath y Sundaram (2015) \\
\hline Chlorella pyrenoidosa & 10 & $0.26 \mathrm{gL}^{-1} \mathrm{~d}^{-1}$ & Erlenmeyer $800 \mathrm{~mL} 25^{\circ} \mathrm{C} . \mathrm{I}_{0}: 180 \mu \mathrm{mol} \mathrm{m}^{2} \mathrm{~s}^{-1}$ & Tang, Han, Li, Miao y Zhong (2011) \\
\hline Chlorella kessleri & 0.04 & $90 \%$ & VTP 4L & Greque y Vieira (2007a) \\
\hline Chlorella vulgaris & 1 & $0.011 \mathrm{gL}^{-1} \mathrm{~d}^{-1}$ & $\begin{array}{l}\text { FBR de membrana con chaqueta de agua } 5,6 \\
L I_{0}: 10800 \mathrm{~lx}\end{array}$ & Fan et ál. (2007) \\
\hline Chlorella vulgaris & 0.04 & $60,1 \%$ & VTP 4L & Greque y Vieira (2007a) \\
\hline Chlorella vulgaris & 2 & $0.15 \mathrm{~g} /\left(\mathrm{L}^{*} \mathrm{~d}\right)$ & $\begin{array}{l}\text { Reactor cilíndrico vertical } 2 \mathrm{~L} \\
\text { Con camisa de agua }\end{array}$ & Naderi, Tadé y Znad, 2015 \\
\hline Chlorella vulgaris & 10 & $0.22 \mathrm{~g} /\left(\mathrm{L}^{*} \mathrm{~d}\right)$ & Sistema ALMCC. Cátodo $500 \mathrm{~mL}$ & Hu, Zhou y Liu (2016) \\
\hline Chlorella vulgaris & 10 & $0.15 \mathrm{~g} /\left(L^{*} \mathrm{~d}\right)$ & Reactor plano agua de mar & Shabani, Sayadi y Rezaei (2016) \\
\hline Spirulina platensis & 10 & $0.49 \mathrm{~g} /\left(\mathrm{L}^{*} \mathrm{~d}\right)$ & Reactor plano agua de mar & Shabani, Sayadi y Rezaei (2016) \\
\hline
\end{tabular}




\begin{tabular}{|c|c|c|c|c|}
\hline Especie algal & $\begin{array}{c}\% \text { entrada de } \\
\mathrm{CO}_{2}\end{array}$ & Fijación de $\mathrm{CO}_{2}$ & Detalles & Referencia \\
\hline Nannochloropsis oculata & 2 & $47.00 \%$ & CPBR $800 \mathrm{~mL}$ & Chiu et ál. (2009) \\
\hline Botryococcus braunii & 5 & $0.5 \mathrm{~g} /\left(\mathrm{L}^{*} \mathrm{~d}\right)$ & $\begin{array}{l}\text { Fermentador BioFlo } 8 \mathrm{~L}, \mathrm{pH} 7.2 \\
3500 \mathrm{~lx} \text {. Fotoperiodo 12:12 }\end{array}$ & Sydney et ál. (2010) \\
\hline Dunaliella tertiolecta & 5 & $0.27 \mathrm{~g} /\left(\mathrm{L}^{*} \mathrm{~d}\right)$ & $\begin{array}{l}\text { Fermentador BioFlo } 8 \mathrm{~L}, \mathrm{pH} 7.2 \\
3500 \mathrm{~lx} \text {. Fotoperiodo 12:12 }\end{array}$ & Sydney et ál. (2010) \\
\hline
\end{tabular}

Abreviaturas: a) ALMCC: celda de captura de carbono microbial tipo air-liff; b) CPBR: fotobiorreactor de columna; c) In: intensidad lumínica inicial; d) PBR: fotobiorreactor; e) TPBR: fotobiorreactor tubular; d) VTP: fotobiorreactor tubular vertical 